\title{
USING CAR4AMS, THE BAYESIAN AMS DATA ANALYSIS CODE
}

\author{
V Palonen ${ }^{1} \cdot \mathrm{P}$ Tikkanen $• \mathrm{~J}$ Keinonen \\ P.O. Box 43, Department of Physics, 00014 University of Helsinki, Finland.
}

\begin{abstract}
The Bayesian CAR (continuous autoregressive) model for accelerator mass spectrometry (AMS) data analysis delivers uncertainties with less scatter and bias. Better detection and estimation of the instrumental error of the AMS machine are also achieved. Presently, the main disadvantage is the several-hour duration of the analysis. The Markov chain Monte Carlo (MCMC) program for CAR model analysis, car4ams, has been made freely available under the GPL license. Included in the package is an R program that analyzes the car4ams output and summarizes the results in graphical and spreadsheet formats. We describe the main properties of the CAR analysis and the use of the 2 parts of the program package for radiocarbon AMS data analysis.
\end{abstract}

\section{INTRODUCTION}

An accelerator mass spectrometry (AMS) result consists of an estimate of a rare-isotope concentration and the associated uncertainty. Confident use of AMS results requires that both be reliable. In an AMS experiment, tens of unknown and some standard samples are measured sequentially. A measurement consists of several determinations of the ratio of a rare isotope and an abundant isotope. The results of the unknown samples are compared to the results of the standards.

AMS laboratories use different methods of data analysis. AMS machine manufacturers deliver software for AMS data analysis and relatively few laboratories have published their methods of data analysis. The chosen method of data analysis can have a significant impact on the quality of the results (Palonen et al. 2008). The commonly used standard error of the mean (sdom) varies randomly and the uncertainties given can be too small or too large by a factor of 2 . In addition, on average the sdom is too small. It has also been shown that, even when the measurements are drawn from a Gaussian distribution, the results based on the sdom are not Gaussian (Palonen et al. 2008). Hence, the use of sdom is the reason for some of the outliers observed in the field. For simplicity, radiocarbon intercomparisons assume a Gaussian distribution for the results even though this is known not to be the case.

We have developed the CAR (continuous autoregressive) model, a Bayesian alternative for AMS data analysis (Palonen and Tikkanen 2007; Palonen 2008; Palonen et al. 2008a). By explicitly using individual counting statistical uncertainties and fitting an overall instrumental error process to the observed scatter of all measurements, the quality of the AMS results can be significantly improved (Palonen et al. 2008b). We present a brief outline of the model and its benefits for the user. We then describe the available CAR modeling software car4ams and how one can start using it. The software and a detailed manual have been made publicly available at http://beam.acclab.helsinki.fi/ vpalonen/car4ams/.

\section{MAIN IDEAS BEHIND THE MODEL}

We represent the individual ${ }^{14} \mathrm{C} /{ }^{13} \mathrm{C}$ or ${ }^{14} \mathrm{C} /{ }^{12} \mathrm{C}$ ion current ratios with $\mathbf{R}=\left(R_{1}, R_{2}, \ldots, R_{N}\right) . M$ different samples are measured. The number of individual measurements made on any particular sample varies.

\footnotetext{
${ }^{1}$ Corresponding author. Email: vesa.palonen@helsinki.fi.

(C) 2010 by the Arizona Board of Regents on behalf of the University of Arizona Proceedings of the 20th International Radiocarbon Conference, edited by A J T Jull RADIOCARBON, Vol 52, Nr 2-3, 2010, p 948-952
} 
The inference of the unknown radiocarbon concentrations $\mathbf{O}=\left(O_{1}, \ldots, O_{M}\right)$ and associated uncertainties is the goal of the analysis. For standard samples, the isotope concentration is known and hence the corresponding $O$ parameter has, for example, a Gaussian prior with $0.1 \% \mathrm{pMC}$ uncertainty.

Two kinds of uncertainties (errors) are assumed for each measurement: known counting statistical uncertainty and unknown instrumental uncertainty. Counting uncertainty is represented with the appropriate Poisson distribution based on the stable isotope current and ${ }^{14} \mathrm{C}$ counting time. The instrumental uncertainty is described with a continuous autoregressive (CAR) process, the parameters of which are inferred from the whole measurement day. The process is capable of describing uncorrelated (white) noise, highly correlated (red) noise (random walk), and moderately correlated (purple) noise in continuous time.

The standard reference level $\mathbf{L}=\left(L_{1}, L_{2}, \ldots, L_{N}\right)$ incorporates the instrumental uncertainty and is described with the CAR process $x(t)$ around a mean $m$ :

$$
L_{i}=x\left(t_{i}\right)+m
$$

For $x\left(t_{i}\right)$, with $\Delta t_{i}=t_{i}-t_{i-1}$, we have

$$
x\left(t_{i}\right)=x\left(t_{i-1}\right) e^{-\alpha \Delta t_{i}}+v_{i}, \text { with } v_{i} \sim N\left(0, \frac{1-e^{-2 \alpha \Delta t_{i}}}{2 \alpha} \sigma^{2}\right)
$$

The parameter $\sigma>0$ describes the strength of the changes in the CAR process per unit time. The correlation parameter $\alpha>0$ describes the amount of correlation ( $\alpha$ is small for a highly correlated process).

Denoting the duration of a ${ }^{14} \mathrm{C}$ measurement with $\tau_{i},{ }^{14} \mathrm{C}$ charge state with $q$, and ${ }^{12} \mathrm{C}$ or ${ }^{13} \mathrm{C}$ current with $I_{i}$, the expected number of counts in a single measurement is $\lambda_{i}=O_{n(i)} L_{i} \frac{I_{i} \tau_{i}}{q e}$ and the measured number of counts is $c_{i}=R_{i} \frac{I_{i} \tau_{i}}{q e}$.

Using uniform priors for simplicity, the joint posterior probability density for the concentrations and the standard reference level is then given by

$$
\begin{aligned}
& p(\alpha, \sigma, m, \mathbf{O}, \mathbf{x} \mid \mathbf{R}) \propto \prod_{s} \exp \left(\frac{-\left(O_{s}-F_{s}\right)^{2}}{2 \sigma_{s}^{2}}\right) \prod_{j=1}^{N} e^{-\lambda_{j}} \lambda_{j}^{c_{j}} \\
& \times \prod_{i=1}^{N} \frac{\sqrt{\alpha}}{\sigma \sqrt{1-e^{-2 \alpha \Delta t_{i}}}} \exp \left(\frac{-\alpha\left(x\left(t_{i}\right)-e^{-\alpha \Delta t_{i}} x\left(t_{i-1}\right)\right)^{2}}{\left(1-e^{-2 \alpha \Delta t_{i}}\right) \sigma^{2}}\right)
\end{aligned}
$$

where the first product represents the priors for the standards, the second product the Poisson distribution of each measurement, and the third product the pdf of the CAR process. The practical inference is done by sampling a chain of points distributed as the above posterior with Markov chain Monte Carlo (MCMC) methods.

\section{BENEFITS OF CAR}

A comparison of different data analysis methods can be done in a frequentist framework, e.g. using the bias of the point estimates from each method (Palonen et al. 2008), or in the Bayesian framework, by comparing the height of probability densities at the true value (Palonen et al. 2008). Hence, 
one or the other method has to be forced to a framework foreign to it. The Bayesian comparison is slightly problematic for frequentist methods because the results have to be interpreted as probabilities for the current sample (instead of frequencies of occurrence in the limit of infinite identical samples). This seems not to be too severe, because in practice they are mostly interpreted as probabilities anyway. The frequentist use of bias is somewhat more problematic because bias is not invariant under a change of parametrization, the type of bias (difference, squared difference, etc.) selected will determine much of the outcome, and the best point estimate of a pdf depends on what kinds of differences the user wants to minimize (Jaynes 2003). Fortunately, the CAR model gives better results in simulated comparisons in both frameworks.

In particular, the sampling-based random scatter of the uncertainty estimate is minimized and the problems in the use of the standard error of the mean (the general negative bias, the negative bias due to correlations, and Student's $t$ phenomena) can be avoided. Full probability distribution functions (pdf) can be used throughout the analysis, thereby removing the risks of assuming an incorrect shape for the final pdf.

Of practical importance to the AMS laboratory is that the type and magnitude of the instrumental error for each measurement day are obtained much more accurately, enabling better recording of the overall performance of the AMS system. This is possible basically because not only the standards but also the unknowns are used in the inference of the instrumental error. The consistency of the results from the same unknown cathode/sample and the known counting error of each measurement are used to infer the additional error needed to explain the results.

Immediate benefits of the CAR model include stable uncertainties, sensitive detection of instrumental error, and default usage of the Poisson distribution. This is especially handy with very old samples. Long-term benefits are more reliable uncertainties, more Gaussian results, fewer outliers, and slightly more accurate results. (Probability densities are higher at true value; $z$ scores are lower.)

The disadvantages are longer time of analysis (several hours), online analysis during measurements not supported, and the same error process for the whole measurement is assumed. Change points are not currently supported. Significant retuning of the system may require separate CAR runs for the different data sets.

\section{STAGES OF CAR4AMS ANALYSIS}

This section details the use of the software implementation of the CAR model, the car4ams program package. We assume that the reader is somewhat familiar with ${ }^{14} \mathrm{C}$ calculations, the details of which can be found in the references (Stuiver and Polach 1977; Donahue et al. 1990; Tuniz et al. 1998; Palonen 2008).

The analysis of AMS measurement-day data is done in 4 steps, explained in detail below:

1. Apply $\delta^{13} \mathrm{C}$ and other known corrections on each measured ratio prior to CAR analysis.

2. Make a Ratios.in file of the data and check the options file (car4ams.in).

3. Run car4ams to get a chain of points drawn from the posterior probability density.

4. Summarize car4ams output and perform background correction with cAnalyze.R (an R script). 


\section{$\delta^{13} \mathrm{C}$ Correction}

Correction for known fractionation is done prior to the CAR analysis both for the standards and the unknown samples (Palonen 2008). The measured ratios $R_{i}$ and stable isotope currents $I_{i}$ are transformed by

$$
\begin{aligned}
& R_{i}^{*}=L_{13, i}^{-1} R_{i}, \text { and } \\
& I_{i}^{*}=L_{13, i} I_{i},
\end{aligned}
$$

where the symbols marked with an asterisk are forwarded to the model, and where, for ${ }^{14} \mathrm{C} /{ }^{13} \mathrm{C}$ measurements,

$$
L_{13, i}=\frac{1+\left(\delta^{13} C\right)_{i} / 1000}{1-25 / 1000}
$$

and, for ${ }^{14} \mathrm{C} /{ }^{12} \mathrm{C}$ measurements,

$$
L_{13, i}=\left(\frac{1+\left(\delta^{13} C\right)_{i} / 1000}{1-25 / 1000}\right)^{2}
$$

Presently, car4ams does not include inference of outlier measurements. If clearly present, outliers should be removed prior to analysis. Some AMS machines may require additional corrections. These should also be performed with the $\delta^{13} \mathrm{C}$ corrections prior to car4ams analysis.

\section{Necessary Input Files}

Measurement data is given as a text file Ratios.in, where each measured ${ }^{14} \mathrm{C} /{ }^{13} \mathrm{C}$ or ${ }^{14} \mathrm{C} /{ }^{12} \mathrm{C}$ ratio is given on 1 line. The input file car4ams.in gives the number of cathodes, sets the priors for each parameter, and contains options for the MCMC sampler. Usually, the same car4ams.in file can be used for all analyses. Note, however, that the standard samples have different priors, and, if the cathode positions of the standards have changed, car4ams.in has to be changed accordingly. The necessary form of the measurement data in Ratios.in and the description of the options of car4ams.in is given in detail in Palonen et al. (2009).

\section{Running car4ams}

The car4ams program is used to compute the probability distributions for all CAR model parameters. The probabilities are given as a group of parameter space points distributed according to the joint posterior probability of all the parameters (Equation 3). The sequence of points is called a MCMC chain. Then, car4ams prints out the MCMC chain to the standard output, which is redirected to a file. The program is run from the command line with

$$
\text { car4ams }>\text { c.txt }
$$

where the last part of the command redirects the chain to the c.txt file. The MCMC chain (the file c.txt) from car4ams represents the joint posterior probability distribution for the parameters. This distribution contains all current information about the parameters. For the more practical part of drawing probability plots and for computing relevant point estimates and uncertainties, an $\mathrm{R}(\mathrm{R}$ Development Core Team 2008) script (cAnalyze.R) is provided. 


\section{Summarizing the Posterior with cAnalyze.R}

The cAnalyze.R script summarizes the joint posterior pdf given by car4ams by calculating point estimates, uncertainties, and various plots. The script cAnalyze. $\mathrm{R}$ is run in $\mathrm{R}$ by giving the command source('cAnalyze.R'). The present version of car4ams does not support background correction as a part of the inference (Palonen 2008). Background correction is performed after analysis in the cAnalyze.R script and is based either on a measured background sample or a constant background level.

Output is given as 2 files. cReport.pdf will contain density plots and other graphical presentations that the user has enabled in the script options. The spreadsheet file cResults.csv will contain the medians, the central posterior intervals, and a symmetric uncertainty estimate (an interval with upper and lower limits at equal distance from the median) for the parameters. ${ }^{14} \mathrm{C}$ ages and uncertainties are given for the parameters representing the ${ }^{14} \mathrm{C}$ concentrations of the cathodes. If background correction is disabled, the output will be directed to the files cReportNoBGC.pdf and cResultsNoBGC.csv.

\section{CONCLUSION}

This paper describes the car4ams program, the software implementation of the CAR model for AMS data analysis. The program is now suited for routine analysis of AMS measurements and it is hoped that the field will benefit from it. In the future, we plan to incorporate the following features: convergence diagnostics; outlier detection; and automatic detection and accounting of ion-source memory effects.

\section{REFERENCES}

Donahue DJ, Linick TW, Jull AJT. 1990. Isotope-ratio and background corrections for accelerator mass spectrometry radiocarbon measurement. Radiocarbon 32(2):135-42.

Jaynes ET. 2003. Probability Theory: The Logic of Science. Cambridge: Cambridge University Press.

Palonen V. 2008. Accelerator mass spectrometry and bayesian data analysis [PhD dissertation]. University of Helsinki Report Series in Physics HU-P-D148. Helsinki: University of Helsinki, Department of Physics. URL: http://urn.fi/URN:ISBN:978-952-10-3262-2.

Palonen V, Tikkanen P. 2007. Pushing the limits of AMS radiocarbon dating with improved Bayesian data analysis. Radiocarbon 49(3)1261-72.

Palonen V, Tikkanen P, Keinonen J. 2008a. A Bayesian measurement model; reliable uncertainties and control over instrumental drift. Journal of Physics D: Applied Physics 41:212001, doi:10.1088/0022-3727/41/21/ 212001.
Palonen V, Tikkanen P, Keinonen J. 2008b. Improving AMS uncertainties and detection of instrumental error. Nuclear Instruments and Methods in Physics Research B 268(7-8):972-5.

Palonen V, Tikkanen P, Keinonen J. 2009. car4ams: a program for Bayesian analysis of accelerator mass spectrometry data [program and manual]. Available at URL: $\quad$ http://beam.acclab.helsinki.fi/ vpalonen/ car4ams/.

$\mathrm{R}$ Development Core Team 2008. R: A Language and Environment for Statistical Computing. Vienna: R Foundation for Statistical Computing. ISBN 3900051-07-0. URL: http://www.r-project.org.

Stuiver M, Polach HA. 1977. Discussion: reporting of ${ }^{14} \mathrm{C}$ data. Radiocarbon 19(3):355-63.

Tuniz C, Bird J, Fink D, Herzog GF. 1998. Accelerator Mass Spectrometry: Ultrasensitive Analysis for Global Science. Boca Raton: CRC Press. 400 p. 